
\title{
3 Research Square \\ Fecal transplants from aged mice impair cognitive function of younger mice
}

\author{
Alfonsina D'Amato \\ Lorenzo Di Cesare-Mannelli \\ Elena Lucarini \\ Angela L. Man \\ Gwenaelle Le Gall \\ Jacopo J. V. Branca \\ Carla Ghelardini \\ Amedeo Amedei \\ Eugenio Bertelli \\ Mari Regoli \\ Alessandra Pacini \\ Giulia Luciani \\ Pasquale Gallina \\ Annalisa Altera \\ Arjan Narbad \\ Massimo Gulisano \\ Lesley Hoyles \\ David Vauzour \\ Claudio Nicoletti
}

\section{Video Byte}

Keywords: Microbiome, gut-brain axis, microbiota, gut microbiome, fecal microbiota transplant, FMT, central nervous system, CNS, hippocampus, aging, proteomics, learning, memory, synaptic plasticity, neurotransmission, short-chain fatty acids, SFCAs, microglia, cognitive function, Barnes maze test

Posted Date: November 12th, 2020

DOI: https://doi.org/10.21203/rs.3.rs-106708/v1

License: (c) (1) This work is licensed under a Creative Commons Attribution 4.0 International License. Read Full License 


\section{Abstract}

A new study suggests that transferring gut microbes from aged to young adult mice has measurable effects on parts of the central nervous system, highlighting the importance of the gut-brain axis in aging. Researchers performed fecal transplants from aged or age-matched donors to younger adult mice. The two groups showed significant differences in their microbial profiles. After transplantation, young adult recipients showed no significant changes in markers of anxiety, explorative behavior, or locomotor activity. But recipients did show impaired spatial learning and memory, as measured by a maze test. These changes were paralleled by alterations in the expression of proteins associated with synaptic plasticity and neurotransmission and changes in microglial cells in the hippocampus - the learning and memory center of the brain. While it remains to be seen whether transplantation from young donors can restore cognitive function in aged recipients, the findings demonstrate that age-related shifts in the gut microbiome can alter components of the central nervous system, pointing to the gut microbiome as one route to combating cognitive decline in the elderly. 